\title{
TERT rs2736100 genotypes are associated with differential risk of myeloproliferative neoplasms in Swedish and Chinese male patient populations
}

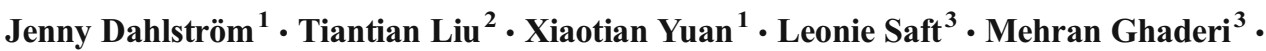 \\ Ya Bin $\mathrm{Wei}^{4} \cdot$ Catharina Lavebratt ${ }^{4} \cdot{\text { Ping } \mathrm{Li}^{5} \cdot \text { Chengyun Zheng }}^{6}$. \\ Magnus Björkholm ${ }^{1}$ - Dawei $\mathrm{Xu}^{1}$
}

Received: 2 August 2016/Accepted: 3 August 2016/Published online: 25 August 2016

(C) The Author(s) 2016. This article is published with open access at Springerlink.com

\begin{abstract}
The telomerase reverse transcriptase (TERT) gene rs2736100 C allele has recently been shown to be associated with an increased risk for myeloproliferative neoplasms (MPNs) among Caucasians. However, it is unknown if this association is present in other ethnical populations and whether rs2736100 allele frequencies mirror the incidence of MPNs in a population. Here we genotyped TERT rs2736100 variants in 126 Swedish and 101 Chinese MPN patients and their age-, sex-, and ethnically-matched healthy controls. Healthy Chinese adults had a higher frequency of the A allele and lower frequencies of the $\mathrm{C}$ allele compared to Swedish counterparts (57.4 vs $47.0 \%$ for A, 42.6 vs $53.0 \%$ for $\mathrm{C}, P=0.006$ ). Both Swedish and Chinese patients harbored significantly higher $\mathrm{C}$ allele frequency than their controls (62.7 vs $53.0 \%$ and 57.4 vs
\end{abstract}

Jenny Dahlström and Tiantian Liu contributed equally to this work.

Jenny Dahlström

Jenny.Dahlstrom@ki.se

Tiantian Liu

liu.tiantian@sdu.edu.cn

1 Department of Medicine, Division of Hematology and Center for Molecular Medicine (CMM), Karolinska Institutet, Karolinska University Hospital Solna, Stockholm 17177, Sweden

2 Department of Pathology, Shandong University School of Medicine, Jinan, China

3 Department of Oncology and Pathology, Karolinska Institutet, Stockholm, Sweden

4 Department of Molecular Medicine and Surgery, Neurogenetics Unit, Karolinska Institutet, Stockholm, Sweden

5 Shandong University School of Nursing, Jinan, China

6 Department of Hematology, The Second Hospital of Shandong University, Jinan, China
$42.6 \%$ for Swedish and Chinese, respectively, $P=0.004$ ). Swedes and Chinese bearing the $\mathrm{CC}$ genotype had a significantly increased risk of MPN compared to AA carriers $(\mathrm{OR}=2.47$; $95 \%$ CI: $1.33-4.57, P=0.003$, for Swedes, and $\mathrm{OR}=3.45$; $95 \%$ CI: $1.52-7.85, P=0.005$, for Chinese). Further analyses showed that rs $2736100 \mathrm{CC}$ was associated with robustly enhanced risk in males only $(\mathrm{CC}$ vs $\mathrm{AA}, \mathrm{OR}=5.11 ; 95 \% \mathrm{CI}$ : $2.19-11.92, P<0.0001)$. The CC-carrying MPN patients exhibited significantly higher TERT expression than patients with the AC genotype. Collectively, the rs 2736100 C is a risk allele for MPNs in Swedish and Chinese males, and the lower incidence of MPNs in the Chinese population is correlated with a lower rs2736100_C risk allele frequency.

Keywords Telomerase · Myeloproliferative neoplasms · Cancer genetics $\cdot$ Single nucleotide polymorphism $\cdot$ TERT

\section{Introduction}

Philadelphia chromosome negative myeloproliferative neoplasms (MPNs), consisting of polycythemia vera (PV), essential thrombocythemia (ET), and primary myelofibrosis (PMF), are characterized by hyperproliferation in the bone marrow leading to an excessive amount of mature cells from one or more of the myeloid lineages [1]. MPNs generally have a relatively indolent course, representing an early stage of leukemogenesis. However, a subset of patients eventually evolves into myelodysplastic syndrome (MDS) or acute myeloid leukemia (AML) [1-3]. Several genetic alterations have been identified in MPNs, with the most common being the JAK2 ${ }^{\mathrm{V} 617 \mathrm{~F}}$ mutation seen in $95 \%$ of PV and $50 \%$ of ET and PMF. Other genetic aberrations include calreticulin (CALR) and thrombopoetin receptor (MPL) mutations that are present in 
70 and $10 \%$ of JAK2 ${ }^{\mathrm{V} 617 \mathrm{~F}}$-negative ET and PMF, respectively $[1,4-6]$. The identification of these mutations provides profound insights into the pathogenesis of MPNs, but the exact etiology remains incompletely understood. For instance, the MPN incidence varies significantly dependent on geographical areas, from approximately $2 / 100,000$ person-years in China [7] to 5.8/100,000 person-years in Europe [1, 8], which highlights differences in genetic susceptibility. However, little is known about genetic events underlying this difference in incidence between Caucasians and Chinese.

Telomerase, a RNA-dependent DNA polymerase with telomerase reverse transcriptase (TERT) as its key catalytic component, is silent in most normal human cells due to the transcriptional repression of the TERT gene, whereas TERT induction coupled with telomerase activation is required for malignant transformation $[9,10]$. The aberrant TERT expression confers malignant cells infinite proliferative potential. Similarly, high telomerase activity and TERT expression has been observed in MPNs [11, 12]. More recently, a number of studies uncovered an association between the rs $2736100 \mathrm{~A}>\mathrm{C}$ single nucleotide polymorphism (SNP) in the TERT gene and the risk of developing MPNs [13-17]. The rs2736100 is located at intron 2 of the TERT locus, and its CC genotype was previously observed to enhance TERT transcription, thereby increasing cancer risk [18]. However, so far, all the data of the rs2736100 association with MPN risk have been exclusively obtained from the analysis of Caucasians. It is currently unclear whether this is the case in the Chinese population or if there is a racial disparity. Moreover, given the significant difference in MPN incidence between Western and Chinese populations, it is important to determine whether the distribution of rs2736100 genotypes differs between these two ethnic groups. In the present study, we aimed to address these issues by genotyping rs 2736100 in both Swedish and Chinese MPN patients and their corresponding healthy controls.

\section{Materials and methods}

\section{Study populations}

One hundred one Chinese MPN patients and 101 age- and sex-matched healthy adults were recruited from Shandong University Hospitals, China. Patients diagnosed with MPN $(N=126)$ at the Karolinska University Hospital in Solna, Stockholm, were also recruited to this study. The SNP rs2736100 has recently been studied in a Swedish general population [19], and an age- and sex-matched part of this population $(n=756)$ was used as control for the Swedish MPN patients. The study was approved by the Shandong University Second Hospital Ethics Committee and Stockholm Regional Ethics Review Board, and informed consent was obtained from all the participants.

\section{DNA extraction and SNP genotyping}

DNA was extracted from blood derived from Swedish and Chinese MPN patients, and Chinese healthy controls using QIAmp DNA blood kit (Qiagen, Hilden, Germany). For Swedish healthy controls, saliva was collected and DNA extracted using a whole-saliva collection device (Oragene•DNA sample collection kit; DNA Genotek Inc., Canada) [19]. DNA concentration was measured on a NanoDrop 2000 spectrophotometer (Thermo Scientific, Waltham, MA, USA). Genotyping of rs2736100 was performed using pre-designed TaqMan SNP genotyping assay kits on a QuantStudio 7 flex system (Applied Biosystems, Waltham, MA, USA) as previously described [20]. The assay included negative controls and was run with the following condition: $95^{\circ} \mathrm{C}$ for $10 \mathrm{~min}$ followed by 40 cycles of $92^{\circ} \mathrm{C}$ for $15 \mathrm{~s}$ and $60^{\circ} \mathrm{C}$ for $1 \mathrm{~min}$. The genotyping success rate was $>95 \%$.

\section{RNA isolation and quantitative reverse transcription-PCR (qRT-PCR)}

Total cellular RNA in granulocytes from MPN patients was isolated using TRIzol reagent (Life Technologies, Carlsbad, CA, USA). Two micrograms of RNA was used for reverse transcription using M-MLV (Life Technologies, Carlsbad, CA, USA)) according to the manufacturer's recommendation. Real-time amplification was performed in triplicate using SYBR Green PCR Master Mix (Life Technologies, Carlsbad, CA, USA) with QuantStudio 7 Flex Teal-Time PCR system (Applied Biosystems, Waltham, MA, USA), and the following primers were used: TERT, 5'-CGGAAGAGTGTCTG GAGCAA-3' (forward) and 5'-GGATGAAGCGGAGT CTGGA-3' (reverse); $\beta 2$-M, 5'-GAATTGCTATGTGTCT GGGT-3' (forward) and 5'-CATCTTCAAACCTCCATGAT G-3' (reverse). Levels of TERT messenger RNA (mRNA) were calculated from threshold cycle values and normalized to $\beta 2-\mathrm{M}$ mRNA abundance, and expressed as arbitrary units.

\section{Telomere length analysis with flow-FISH}

Telomere length in granulocytes from the Swedish MPN patients was measured using flow-FISH as previously described [21]. In short, fluorescent PNA probes (Panagene, Daejeon, Korea) were hybridized to the telomere sequence and the fluorescent signal was measured using a Gallios flow cytometer (Beckman Coulter, Brea, CA, USA) and analyzed using the Kaluza software (Beckman Coulter, Brea, CA, USA). Fluorescent MESF-FITC beads (Bangs Laboratories, Fishers, IN, USA) were used and the fluorescent signal was quantified using the QuickCal v.2.3 data analysis program (Bangs Laboratories, Fishers, IN, USA). 


\section{Statistical analysis}

Age and sex differences between patients and controls were compared using Mann-Whitney $U$ and Fisher's exact test, respectively. Differences in telomere length and TERT mRNA levels among different genotype groups were determined using Mann-Whitney $U$ test. Fisher's exact test was used to determine odds ratio with $95 \%$ confidence interval (CI) and $P$ value. All statistical analyses were performed in GraphPad Prism. $P$ values $<0.05$ were considered statistically significant.

\section{Results}

\section{Demographic and clinical characteristics of study subjects}

A total of 126 MPN patients from Sweden and 101 from China were genotyped for rs2736100 variants. Clinical features including age, sex, MPN subtype, and mutation status (JAK2 ${ }^{\mathrm{V} 617 \mathrm{~F}}$ and CALR mutations) are summarized in Table 1. Seven hundred fifty-six Swedish and 101 Chinese healthy age- and sex-matched adults were used as controls (Table 1). ET were more common and PV more uncommon among the Chinese patients than the Swedish patients. The Chinese patients were also younger than the Swedish patients.

\section{Different rs2736100 allele distributions in healthy Swedish and Chinese populations}

We first compared the distribution of rs2736100 genotypes in the healthy Swedish and Chinese control populations. As shown in Table 2, the Chinese population had lower $\mathrm{C}$ and higher A allele frequencies than the Swedish population (42.6 vs $53.0 \%$ for $\mathrm{C}$ and 57.4 vs $47.0 \%$ for A, respectively, $P=$ $0.006)$. To verify whether this finding was representative, we further collected published genotyping data from another cohort of 289 healthy adults from the Shandong area (north) [22] and one from south of China (Shanghai and Guangzhou areas) [18]. Both of them showed a rs2736100 genotype distribution very similar to that of the present study (Table 3). The rs2736100 allele/genotype distribution in healthy populations from Sweden and other European countries (south and east) $[15,16]$ was also compared to the healthy populations in China. All of the previously published studies displayed largely similar rs 2736100 variant frequency, with significantly higher $\mathrm{C}$ while lower A alleles in European countries (48.0 vs $57.4 \%$ and 52.0 vs $42.6 \%$ for A and C, respectively, $P<0.001)$. There was no difference in the AC genotype between Chinese and Caucasians (Tables 2 and 3).
Table 1 Characteristics of healthy controls and patients with MPN

\begin{tabular}{|c|c|c|c|c|}
\hline & \multicolumn{2}{|l|}{ Sweden } & \multicolumn{2}{|l|}{ China } \\
\hline & Controls & MPN & Controls & MPN \\
\hline Number & 756 & 126 & 101 & 101 \\
\hline \multicolumn{5}{|l|}{ Age (years) } \\
\hline Mean $\pm \mathrm{SD}$ & $64 \pm 5$ & $64 \pm 14$ & $58 \pm 15$ & $58 \pm 15$ \\
\hline Median (range) & $64(54-74)$ & $65(25-106)$ & $60(17-82)$ & $60(17-82)$ \\
\hline Sex [\%females] & 53 & 53 & 50 & 50 \\
\hline \multicolumn{5}{|l|}{ MPN subtype, $n(\%)$} \\
\hline PV & & $41(32.5)$ & & $16(15.8)$ \\
\hline ET & & $40(31.7)$ & & 38 (37.6) \\
\hline PMF & & $28(22.3)$ & & $15(14.9)$ \\
\hline MPN-NOS & & $17(13.5)$ & & $32(31.7)$ \\
\hline \multicolumn{5}{|l|}{ JAK2-status, $n(\%)$} \\
\hline JAK2 V617F & & $60(47.6)$ & & $38(37.6)$ \\
\hline JAK2 V617F ${ }^{+}$ & & $66(52.4)$ & & $56(55.4)$ \\
\hline Unknown & & 0 & & $7(7.0)$ \\
\hline CALR mutation & & 45 & & Unknown \\
\hline
\end{tabular}

$M P N$ myeloproliferative neoplasm, $P V$ polycythemia vera, $E T$ essential thrombocythemia, $P M F$ primary myelofibrosis

\section{Rs2736100_C allele association with risk of MPN}

Given different distributions of the rs2736100 allele frequency between control populations from two different countries, we chose to analyze the Swedish and Chinese MPN patients separately. The rs2736100_C allele frequency was significantly higher in both Swedish and Chinese MPN patients than in their corresponding controls (62.7 vs $53.0 \%$ and 57.4 vs $42.6 \%$ for Swedish and Chinese patients, respectively, $P=0.004$ ) (Table 4). Compared to the AA variant carriers, Swedish and Chinese patients bearing the $\mathrm{CC}$ genotype exhibited significantly increased

Table 2 Comparison of genotype distribution of TERT rs2736100 in healthy control populations in Sweden and China

\begin{tabular}{|c|c|c|c|c|}
\hline$r s 2736100$ & & $\begin{array}{l}\text { Sweden } \\
\mathrm{N}(\%)\end{array}$ & $\begin{array}{l}\text { China } \\
\text { N (\%) }\end{array}$ & $P$ value \\
\hline \multirow[t]{7}{*}{ Genotype } & & $756(100)$ & $101(100)$ & \\
\hline & $\mathrm{AC}+\mathrm{CC}$ & $589(77.9)$ & $68(67.3)$ & \multirow[t]{2}{*}{0.024} \\
\hline & AA & $167(22.1)$ & $33(32.7)$ & \\
\hline & $\mathrm{AA}+\mathrm{CC}$ & $379(50.1)$ & $51(50.5)$ & \multirow[t]{2}{*}{0.916} \\
\hline & $\mathrm{AC}$ & 377 (49.9) & $50(49.5)$ & \\
\hline & $\mathrm{AA}+\mathrm{AC}$ & $544(72.0)$ & $83(82.2)$ & \multirow{2}{*}{0.031} \\
\hline & $\mathrm{CC}$ & $212(28.0)$ & $18(17.8)$ & \\
\hline \multirow[t]{2}{*}{ Allele } & A & $711(47.0)$ & $116(57.4)$ & \multirow[t]{2}{*}{0.006} \\
\hline & $\mathrm{C}$ & $801(53.0)$ & $86(42.6)$ & \\
\hline
\end{tabular}

OR and P-value generated using Fishers' exact test

Significant $\mathrm{p}$-values are shown in italic 
Table 3 Published rs2736100 genotype distributions of healthy populations in China and Europe

\begin{tabular}{|c|c|c|c|c|c|c|c|c|}
\hline Author & Number & $\mathrm{AA}(\%)$ & $\mathrm{AC}(\%)$ & $\mathrm{CC}(\%)$ & $\mathrm{A}(\%)$ & $\mathrm{C}(\%)$ & Area & Reference \\
\hline \multicolumn{9}{|l|}{ China } \\
\hline Dahlström et al. & 101 & $33(32.7)$ & $50(49.5)$ & $18(17.8)$ & $116(57.4)$ & $86(42.6)$ & North $^{\mathrm{a}}$ & This study \\
\hline Yuan et al. & 289 & $86(29.8)$ & $144(49.8)$ & $59(20.4)$ & $316(54.7)$ & $262(45.3)$ & North $^{\mathrm{a}}$ & [18] \\
\hline Wei et al. & 2520 & $814(32.3)$ & $1269(50.4)$ & $437(17.3)$ & $2897(57.5)$ & $2143(42.5)$ & South $^{\mathrm{b}}$ & [16] \\
\hline Total & 2910 & $933(32.1)^{*}$ & $1463(50.3)^{*}$ & $514(17.6)^{*}$ & $3329(57.2)^{*}$ & $2491(42.8)^{*}$ & & \\
\hline \multicolumn{9}{|l|}{ Europe } \\
\hline Dahlström et al. & 756 & $167(22.1)$ & 377 (49.9) & $212(28.0)$ & $711(47.0)$ & $801(53.0)$ & Sweden & This study \\
\hline Jäger et al. & 202 & $47(23.3)$ & $88(43.6)$ & $67(33.2)$ & $182(45.0)$ & $222(55.0)$ & Italy & [14] \\
\hline Krahling et al. & 400 & $111(27.8)$ & $188(47.0)$ & $101(25.2)$ & $410(51.3)$ & $390(48.7)$ & Hungary & [13] \\
\hline \multirow[t]{2}{*}{ Total } & 1358 & $325(23.9)^{* *}$ & $653(48.0)^{* *}$ & $380(28.1)^{* *}$ & $1303(48.0)^{* *}$ & $1413(52.0)^{* *}$ & & \\
\hline & & $\mathrm{AA}$ vs $\mathrm{AC}+\mathrm{CC}$ & $\mathrm{AC}$ vs $\mathrm{AA}+\mathrm{CC}$ & $\mathrm{CC}$ vs $\mathrm{AA}+\mathrm{AC}$ & & C vs A & & \\
\hline$P$ value (* vs $* *)$ & & $<0.001$ & 0.106 & $<0.001$ & & $<0.001$ & & \\
\hline
\end{tabular}

OR and $P$ value were generated using chi-squared test

${ }^{a}$ From Shandong area

${ }^{\mathrm{b}}$ From Shanghai and Guangzhou areas

risk of MPN $(\mathrm{OR}=2.47 ; 95 \%$ CI: $1.33-4.57, P=0.003$, for Swedish, and OR $=3.45 ; 95 \%$ CI: $1.52-7.85, P=0.005$, for Chinese patients) (Table 4). The AC genotype was also associated with an increased risk of MPN, but the $P$ value for Chinese was at borderline significance, likely due to relatively few patients (AC vs AA: $\mathrm{OR}=2.02 ; 95 \%$ CI: $1.00-4.08, P=0.057$, for Chinese, and OR $=1.89 ; 95 \%$ CI: $1.05-3.41, P=0.034$, for Swedish). Higher frequency of $\mathrm{C}$ alleles or $\mathrm{CC}$ genotypes was observed in all MPN subtypes, and there was no significant difference among patients with PV, ET, and PMF, respectively.
rs2736100_C allele association with risk of MPN in males

As shown in Table 5, there was no difference in the rs 2736100 allele distribution between control males and females. However, MPN patients displayed a sex-dependent variant difference: male patients carried significantly more $\mathrm{C}$ alleles than control males did (66.8 vs $52.5 \%, P<0.001)$, and the $\mathrm{AC}$ and $\mathrm{CC}$ genotypes were associated with increased risk of MPNs in males compared to the AA variant $(\mathrm{OR}=3.48$; $95 \%$ CI: $1.53-$ 7.91, $P=0.002$, for AC; OR $=5.11 ; 95 \%$ CI: 2.19-11.92,

Table 4 Comparison of TERT rs2736100 genotypes in MPN patients and healthy controls

\begin{tabular}{|c|c|c|c|c|c|c|c|c|}
\hline \multirow[t]{3}{*}{ rs2736100 genotype } & \multicolumn{4}{|l|}{ Sweden } & \multicolumn{4}{|l|}{ China } \\
\hline & \multirow{2}{*}{$\begin{array}{l}\text { Control } \\
n(\%)\end{array}$} & \multicolumn{3}{|l|}{ MPN } & \multirow{2}{*}{$\begin{array}{l}\text { Control } \\
n(\%)\end{array}$} & \multicolumn{3}{|l|}{ MPN } \\
\hline & & $n(\%)$ & OR $(95 \% \mathrm{CI})$ & $P$ & & $n(\%)$ & OR $(95 \% \mathrm{CI})$ & $P$ \\
\hline All & $756(100)$ & $126(100)$ & & & $101(100)$ & $101(100)$ & & \\
\hline \multicolumn{9}{|l|}{ Alleles } \\
\hline A & $711(47.0)$ & $94(37.3)$ & 1.0 (ref) & & $116(57.4)$ & $86(42.6)$ & 1.0 (ref) & \\
\hline $\mathrm{C}$ & $801(53.0)$ & $158(62.7)$ & $1.49(1.13-1.96)$ & 0.004 & $86(42.6)$ & $116(57.4)$ & $1.82(1.23-2.70)$ & 0.004 \\
\hline \multicolumn{9}{|l|}{ Genotypes } \\
\hline AA & $167(22.1)$ & $15(11.9)$ & 1.0 (ref) & & $33(32.7)$ & $17(16.8)$ & 1.0 (ref) & \\
\hline $\mathrm{AC}$ & 377 (49.9) & $64(50.8)$ & $1.89(1.05-3.41)$ & 0.034 & $50(49.5)$ & $52(51.5)$ & $2.02(1.00-4.08)$ & 0.057 \\
\hline $\mathrm{CC}$ & $212(28.0)$ & $47(37.3)$ & $2.47(1.33-4.57)$ & 0.003 & $18(17.8)$ & $32(31.7)$ & $3.45(1.52-7.85)$ & 0.005 \\
\hline $\mathrm{AA}+\mathrm{AC}$ & $544(72.0)$ & $79(62.7)$ & 1.0 (ref) & & $83(82.2)$ & $69(68.3)$ & 1.0 (ref) & \\
\hline $\mathrm{CC}$ & $212(28.0)$ & $47(37.3)$ & $1.53(1.03-2.27)$ & 0.044 & $18(17.8)$ & $32(31.7)$ & $2.14(1.11-4.14)$ & 0.033 \\
\hline $\mathrm{AC}+\mathrm{CC}$ & $589(77.9)$ & $111(88.1)$ & 1.0 (ref) & & $68(67.3)$ & $84(83.2)$ & 1.0 (ref) & \\
\hline AA & $167(22.1)$ & 15 (11.9) & $0.48(0.27-0.84)$ & 0.009 & $33(32.7)$ & $17(16.8)$ & $0.42(0.21-0.81)$ & 0.014 \\
\hline
\end{tabular}

OR and $P$ value were generated using Fishers' exact test

$M P N$ myeloproliferative neoplasms, $O R$ odds ratio, $C I$ confidence interval

Significant $p$-values are shown in italic 
Table 5 Genotypes of TERT rs2736100 in men and women

\begin{tabular}{|c|c|c|c|c|c|c|c|c|}
\hline \multirow[t]{2}{*}{$r s 2736100$ genotype } & \multicolumn{4}{|l|}{ Men } & \multicolumn{4}{|l|}{ Women } \\
\hline & $\begin{array}{l}\text { Control } \\
n(\%)\end{array}$ & $n(\%)$ & $\begin{array}{l}\text { MPN } \\
\text { OR }(95 \% \text { CI })\end{array}$ & $P$ & $\begin{array}{l}\text { Control } \\
n(\%)\end{array}$ & $n(\%)$ & $\begin{array}{l}\text { MPN } \\
\text { OR }(95 \% \text { CI })\end{array}$ & $P$ \\
\hline All & $405(100)$ & $110(100)$ & & & $452(100)$ & $117(100)$ & & \\
\hline \multirow[t]{2}{*}{ A } & $385(47.5)$ & $73(33.2)$ & 1.0 (ref) & & $442(48.9)$ & $107(45.7)$ & 1.0 (ref) & \\
\hline & $425(52.5)$ & $147(66.8)$ & $1.82(1.33-2.49)$ & $<0.001$ & $462(51.1)$ & $127(54.3)$ & $1.14(0.85-1.52)$ & 0.420 \\
\hline AA & $87(21.5)$ & $7(6.4)$ & 1.0 (ref) & & $113(25.0)$ & $25(21.4)$ & 1.0 (ref) & \\
\hline $\mathrm{AC}$ & $211(52.1)$ & $59(53.6)$ & $3.48(1.53-7.91)$ & 0.002 & $216(47.8)$ & $57(48.7)$ & $1.19(0.71-2.01)$ & 0.601 \\
\hline \multirow[t]{3}{*}{$\mathrm{CC}$} & $107(26.4)$ & $44(40.0)$ & $5.11(2.19-11.92)$ & $<0.0001$ & $123(27.2)$ & $35(29.9)$ & $1.29(0.72-2.28)$ & 0.469 \\
\hline & Control & & & & MPN & & & \\
\hline & Men & & Women & & Men & & Women & \\
\hline $\mathrm{AC}+\mathrm{CC}$ & $318(78.5)$ & $339(74.3)$ & 1.0 (ref) & & 103 (93.6) & $92(78.6)$ & 1.0 (ref) & \\
\hline $\mathrm{AA}$ & $87(21.5)$ & $113(25.7)$ & $0.82(0.60-1.13)$ & 0.227 & $7(6.4)$ & $25(21.4)$ & $0.25(0.10-0.61)$ & 0.001 \\
\hline
\end{tabular}

OR and $P$ value were generated using Fisher's exact test

$O R$ odds ratio, $C I$ confidence interval

$P<0.0001$, for CC) (Table 5). In contrast, no differences in rs2736100 variants were observed between female controls and female patients (Table 5). Within the patient population, men were less likely to harbor the AA variant $(\mathrm{OR}=0.25 ; 95 \% \mathrm{CI}$ : $0.10-0.61, P<0.001)$ whereas no difference was seen in the healthy population (Table 5).

\section{Rs2736100 variant association with JAK2 $2^{\mathrm{V} 617 \mathrm{~F}}$ and CALR mutations in MPNs}

The relationship between major molecular subtypes in MPN (JAK2 ${ }^{\text {V617F }}$ and CALR mutations) and rs2736100 variants was analyzed. The association between CALR and rs2736100 was only studied in the Swedish MPN patients. There was no association between any specific TERT rs2736100 variant and the presence of JAK2 ${ }^{\mathrm{V} 617 \mathrm{~F}}$. Patients harboring CALR mutations (insertion or deletion) $(n=45)$ tended to have a lower frequency of the $\mathrm{CC}$ variant compared to those carrying wt CALR, but the difference did not reach a significant level $(\mathrm{OR}=0.44 ; 95 \%$ CI: $0.20-1.01, P=0.07)$.

\section{The relationship of $\mathbf{r s 2 7 3 6 1 0 0}$ variants with TERT expression and telomere length in MPNs}

TERT mRNA expression and leukocyte telomere length was previously assessed in the Swedish cohort of MPN patients [21], and we thus further determined a potential impact of different rs2736100 genotypes on TERT expression and telomere homeostasis. Patients bearing the $\mathrm{CC}$ genotype expressed the highest levels of TERT mRNA compared to those with AA and $\mathrm{AC}$ variants. There was a significant difference between
AC- and CC-carrying patients $(P=0.024)$. The lowest TERT expression was observed in patients with AA genotype, but the difference was not significant, likely due to too few patients $(N=5)$. In MPN patients there was no correlation between rs2736100 allele variant and telomere length (Fig. 1).

\section{Discussion}

The TERT rs2736100_C allele has recently been identified to be associated with increased risk of MPNs based on analyses of Caucasian patients [13-16]. This finding provides significant insights into genetic susceptibility to MPN. However, a number of issues remain to be defined: first, it is well known that racial disparities exists in both germline and somatic genetic alterations in the pathogenesis of cancer [22]. Thus, it is important to evaluate whether the correlation between rs2736100 allele variant and risk of MPN is also observed in other ethnic populations. Second, it is currently unclear whether the influence of TERT rs2736100_C allele on MPN susceptibility is identical in both males and females. Third, there is a threefold difference in MPN incidence between European countries and China, but the underlying mechanism is unclear. Is there a link between rs2736100 frequency distribution and MPN incidence? Finally, abnormal telomere length and structure is widespread in MPNs $[11,21]$, and it is unknown whether different rs2736100 variants influence TERT expression and telomere length differentially in MPNs. To answer these questions we recruited healthy adults and MPN patients from both Sweden and China for rs2736100 genotyping. The results presented here demonstrate that (i) the 
A)

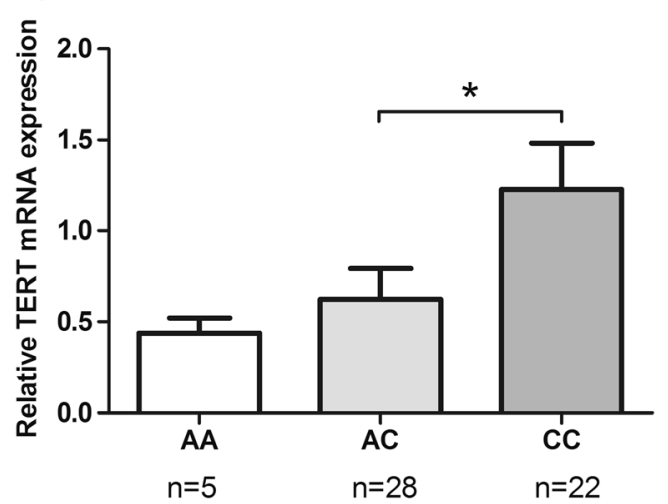

B)

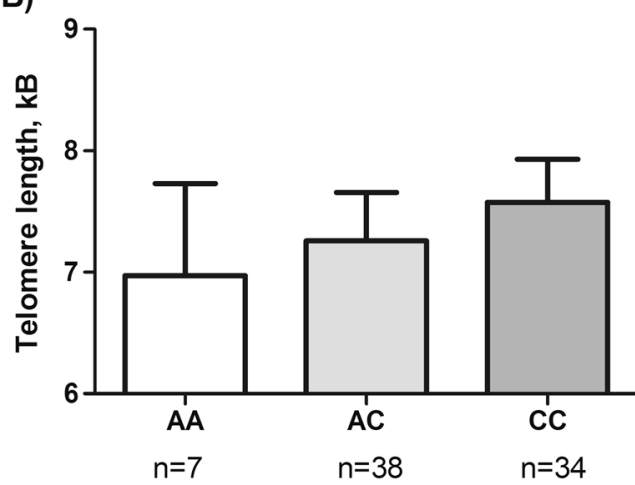

Fig. 1 TERT mRNA expression and telomere length in MPN patients with different TERT rs2736100 variants. Total cellular RNA and genomic DNA were extracted from granulocytes derived from Swedish MPN patients harboring different TERT rs2736100 genetic variants. TERT mRNA and telomere length were measured by using qRT-PCR and flow-FISH, respectively. a TERT mRNA expression and (b) telomere length. $* P<0.05$

rs2736100_C allele is significantly associated with susceptibility to MPNs in both Swedish and Chinese individuals, but the association was restricted to male patients; (ii) the Chinese healthy population bears significantly lower rs2736100_C but higher A alleles than their Swedish counterpart, which is highly consistent with the lower incidence of MPNs in China; (iii) the rs2736100_CC-carrying Swedish patients display highest TERT expression in their myeloid cells.

The accumulated evidence suggests a close connection between germline genetic variation and susceptibility to human diseases. Given TERTs critical role in carcinogenesis, SNPs at the TERT locus have been extensively analyzed for their association with cancer risk, among which rs 2736100 variants are most studied [18, 20, 23-25]. Indeed, the rs2736100_C allele is reported to increase the risk of multiple types of cancer $[18,20,23-25]$. It is believed that the CC genotype promotes TERT transcription, thereby upregulating telomerase activity and maintaining telomere length required for unlimited proliferation of cancer cells [18]. Similarly, high telomerase activity in the bone marrow of patients with MPN has been reported [12]. Meanwhile, telomere shortening is well established in MPN myeloid cells [11, 21, 26], likely due to the hyperproliferation of these cells in MPNs. In this study, we did observe the highest TERT expression in granulocytes derived from MPN patients bearing the $\mathrm{CC}$ genotype. These results indicate that the $\mathrm{C}$ allele may be able to upregulate TERT expression and in turn compensate for overerosion of telomere length in MPNs. In addition, TERT possesses many other important activities independent of its telomerelengthening function [27-30], which has been implicated in cancer development and progression. It is conceivable that these TERT effects may similarly contribute to MPN pathogenesis.

Racial or ethnic disparities in disease incidence and pathogenesis have been well documented, and different genetic backgrounds are believed to play important parts. Likewise, there is a significant difference in the MPN incidence between Han Chinese $(2 / 100,000)$ and European Caucasians $(5.8 / 100,000)$ [8]. However, the underlying mechanism remains poorly understood. In the present study, we found that the rs2736100_C allele contributed to susceptibility to MPNs in both Swedish and Chinese populations. Moreover, the frequency of the A and $\mathrm{C}$ allele was significantly higher and lower, respectively, in Chinese controls than in Swedish and European Caucasian controls. These findings collectively reveal a positive correlation between MPN incidence and the rs 2736100 C allele frequency. As rs2736100_C alleles are associated with an increased risk of many other malignancies [18, 20, 23-25], it is of interest to determine whether the same pattern can be identified in other malignancies where the incidence is lower in China than in Europe. In addition to TERT rs2736100, some other genetic variants have also been identified to be associated with MPN risk [17] and further comparison of their variant frequency between Chinese and Caucasians may contribute to a better understanding of germline variant effects on MPN incidence.

Intriguingly, our results showed a significant association of rs2736100_C with susceptibility to MPNs only in males. This observation is likely unbiased, because there is no difference in the rs2736100 genotype distribution between males and females in the general population in Sweden or China. Information on association between gender and allele variant of TERT rs2736100 in MPN patients is lacking in the majority of published studies [14-16], but one study reported a similar variant distribution in men and women [13]. Among MPN patients, women have been reported to have superior survival compared to men [31, 32]. One study reports that the shorter overall survival seen in male MPN patients is mainly due to increased frequency of secondary AML transformation [32], whereas another states that there are no gender differences in the risk of developing a secondary malignancy [33]. The CC genotype of TERT rs 2736100 has also been associated with poorer response to anticancer agents and with cancerpromoting mutations, such as mutations in P53 [34]. Krahling 
et al. recently reported that MPN patients with the CC genotype had a higher probability to die from secondary solid tumors [15]. Taken together, these data indicate that the adverse outcome seen in male MPN patients may be associated with the rs2736100_CC genotype. Further studies are needed to verify and define the mechanistic role of rs2736100 variants in MPN progression and response to treatment.

We observed that the rs2736100_C allele was evenly distributed in all three subtypes of MPNs (PV, ET, and MF) with different genetic mutations (JAK2 ${ }^{\mathrm{V} 617 \mathrm{~F}}$ or CALR mutations). Similar findings were also documented in publications by Trifa et al. and others $[13,17]$. These data collectively suggest that the $\mathrm{C}$ allele serves as a general risk factor for MPNs with no preferential susceptibility to a specific molecular subtype.

In summary, we demonstrate that the rs $2736100 \_\mathrm{C}$ allele is associated with increased risk to develop MPNs in both Swedish and Chinese populations. The direct comparison of the rs2736100 genotype between Swedish and Chinese healthy individuals shows a higher A but lower $\mathrm{C}$ allele frequency in Chinese individuals, which is correlated with a lower MPN prevalence in China. Further studies are required to dissect a causal relationship between the rs2736100_C allele and MPN development. These studies are needed to define how this allele contributes to MPN pathogenesis and to evaluate its possible role in AML transformation and disease progression.

Acknowledgments This study was supported by grants from the National Basic Research Program of China (grant no. 973 Program 2012CB911202); the National Natural Science Foundation of China (no. 81502409), the Adolf H. Lundin Charitable Foundation, the Swedish Research Council, Swedish Cancer Society, Cancer Society in Stockholm, Stockholm County Council, and Gunnar Grimfors' endowment fund.

Compliance with ethical standards The study was approved by the Shandong University Second Hospital Ethics Committee and Stockholm Regional Ethics Review Board, and informed consent was obtained from all the participants.

Conflict of interest The authors declare that they have no conflict of interest.

Open Access This article is distributed under the terms of the Creative Commons Attribution 4.0 International License (http:// creativecommons.org/licenses/by/4.0/), which permits unrestricted use, distribution, and reproduction in any medium, provided you give appropriate credit to the original author(s) and the source, provide a link to the Creative Commons license, and indicate if changes were made.

\section{References}

1. Michiels JJ, Berneman Z, Schroyens W et al (2015) Changing concepts of diagnostic criteria of myeloproliferative disorders and the molecular etiology and classification of myeloproliferative neoplasms: from Dameshek 1950 to Vainchenker 2005 and beyond. Acta Haematol 133:36-51

2. Björkholm M, Derolf AR, Hultcrantz M et al (2011) Treatmentrelated risk factors for transformation to acute myeloid leukemia and myelodysplastic syndromes in myeloproliferative neoplasms. J Clin Oncol 29:2410-2415

3. Björkholm M, Hultcrantz M, Derolf $\AA$ (2014) Leukemic transformation in myeloproliferative neoplasms: therapy-related or unrelated? Best Pract Res Clin Haematol 27:141-153

4. Nangalia J, Massie CE, Baxter EJ et al (2013) Somatic CALR mutations in myeloproliferative neoplasms with nonmutated JAK2. N Engl J Med 369:2391-2405

5. Langabeer SE, Andrikovics H, Asp J et al (2015) Molecular diagnostics of myeloproliferative neoplasms. Eur J Haematol 95:270-279

6. Klampfl T, Gisslinger H, Harutyunyan AS et al (2013) Somatic mutations of calreticulin in myeloproliferative neoplasms. N Engl J Med 369:2379-2390

7. http://haodf.health.sohu.com/disease/zhenxinghong xibaozengduozheng/jieshao.htm. 2009. Accessed 02 Aug 2016

8. Hultcrantz M, Andersson TM-L, Landgren OM et al (2015) A population-based study of incidence of myeloproliferative neoplasms in Sweden between 2000 and 2012. American society of hematology 57th annual meeting and exposition, Orlando

9. Daniel M, Peek GW, Tollefsbol TO (2012) Regulation of the human catalytic subunit of telomerase (hTERT). Gene 498:135-146

10. Kong F, Zheng C, Xu D (2014) Telomerase as a "stemness" enzyme. Sci China Life Sci 57:564-570

11. Bernard L, Belisle C, Mollica L et al (2009) Telomere length is severely and similarly reduced in JAK2V617F-positive and negative myeloproliferative neoplasms. Leukemia 23:287-291

12. Spanoudakis E, Bazdiara I, Pantelidou D et al (2011) Dynamics of telomere's length and telomerase activity in Philadelphia chromosome negative myeloproliferative neoplasms. Leuk Res 35:459-464

13. Trifa AP, Bănescu C, Tevet $\mathrm{M}$ et al (2016) TERT rs2736100 A > C SNP and JAK2 46/1 haplotype significantly contribute to the occurrence of JAK2 V617F and CALR mutated myeloproliferative neoplasms - a multicentric study on 529 patients. Br J Haematol $174: 218-226$

14. Oddsson A, Kristinsson SY, Helgason H et al (2014) The germline sequence variant rs2736100_C in TERT associates with myeloproliferative neoplasms. Leukemia 28:1371-1374

15. Krahling T, Balassa K, Kiss KP et al (2016) Co-occurrence of Myeloproliferative Neoplasms and solid tumors is attributed to a Synergism between cytoreductive therapy and the common TERT Polymorphism rs2736100. Cancer Epidemiol Biomarkers Prev 25: 98-104

16. Jäger R, Harutyunyan AS, Rumi E et al (2014) Common germline variation at the TERT locus contributes to familial clustering of myeloproliferative neoplasms. Am J Hematol 89:1107-1110

17. Tapper W, Jones AV, Kralovics R et al (2015) Genetic variation at MECOM, TERT, JAK2 and HBS1L-MYB predisposes to myeloproliferative neoplasms. Nat Commun 6:6691

18. Wei R, Cao L, Pu H et al (2015) TERT Polymorphism rs2736100-C is associated with EGFR mutation-positive non-small cell lung cancer. Clin Cancer Res 21:5173-5180

19. Wei YB, Martinsson L, Liu JJ et al (2016) hTERT genetic variation in depression. J Affect Disord 189:62-69

20. Yuan X, Meng Y, Li P, et al (2016) The association between the TERT rs2736100 AC genotype and reduced risk of upper tract urothelial carcinomas in a Han Chinese population. Oncotarget 7: 31972-31979

21. Dahlström J, Zhang X, Ghaderi M et al (2015) Dysregulation of shelterin factors coupled with telomere shortening in Philadelphia chromosome negative myeloproliferative neoplasms. Haematologica 100:e402-e405 
22. Yuan X, Liu C, Wang K, et al (2016) The genetic difference between Western and Chinese urothelial cell carcinomas: infrequent FGFR3 mutation in Han Chinese patients. Oncotarget 7:2582625835

23. Chen XF, Cai S, Chen QG et al (2012) Multiple variants of TERT and CLPTM1L constitute risk factors for lung adenocarcinoma. Genet Mol Res 11:370-378

24. Bojesen SE, Pooley KA, Johnatty SE et al (2013) Multiple independent variants at the TERT locus are associated with telomere length and risks of breast and ovarian cancer. Nat Genet 45:371-384, 384e 371-372

25. Mocellin S, Verdi D, Pooley KA et al (2012) Telomerase reverse transcriptase locus polymorphisms and cancer risk: a field synopsis and meta-analysis. J Natl Cancer Inst 104:840-854

26. Elena C, Rumi E, Portolan M et al (2011) Flow-FISH evaluation of telomere length in Philadelphia-negative myeloproliferative neoplasms. Haematologica 96:1236-1238

27. $\mathrm{Ci} \mathrm{X}, \mathrm{Li} \mathrm{B}, \mathrm{Ma} \mathrm{X}$ et al (2015) Bortezomib-mediated down-regulation of telomerase and disruption of telomere homeostasis contributes to apoptosis of malignant cells. Oncotarget 6:38079-38092

28. Liu Z, Li Q, Li K et al (2013) Telomerase reverse transcriptase promotes epithelial-mesenchymal transition and stem cell-like traits in cancer cells. Oncogene 32:4203-4213
29. Ding D, Xi P, Zhou J et al (2013) Human telomerase reverse transcriptase regulates MMP expression independently of telomerase activity via NF-kB-dependent transcription. FASEB J 27:4375-4383

30. Singhapol C, Pal D, Czapiewski R et al (2013) Mitochondrial telomerase protects cancer cells from nuclear DNA damage and apoptosis. PLoS One 8:e52989

31. Hultcrantz M, Kristinsson SY, Andersson TM et al (2012) Patterns of survival among patients with myeloproliferative neoplasms diagnosed in Sweden from 1973 to 2008: a population-based study. J Clin Oncol 30:2995-3001

32. Stein B, Spivak JL, Moliterno AR (2015) Gender is a core modifier of disease outcomes and survival in the MPN. American Society of Hematology, 57th annual meeting and exposition, Orlando

33. Ravn Landtblom A, Bower H, Andersson TM-L et al (2016) Increased risk of second malignancies in patients with myeloproliferative neoplasms diagnosed in Sweden 1973-2009 - a populationbased cohort study of 9,379 patients. European Hematology Association 21st Congress, Copenhagen

34. Kim J, Jones-Hall YL, Wei R et al (2013) Association between hTERT rs2736100 polymorphism and sensitivity to anti-cancer agents. Front Genet 4:162 